\title{
Factores asociados a hipoglucemia neonatal transitoria en recién nacidos sanos, en el Hospital Universitario San Ignacio, estudio de casos y controles
}

\author{
Case-Control Study Factors Associated with Transient Neonatal \\ Hypoglycemia in Healthy Newborns, at Hospital Universitario San \\ Ignacio
}

Fecha de recepción: 20 Enero 2017 | Fecha de aprobación: 06 Febrero 2017

\author{
Ana María Berttoloto \\ Pontificia Universidad Javeriana, Colombia \\ Yaris Anzully Vargas Vaca \\ Pontificia Universidad Javeriana, Colombia \\ Paula Carolina Guzmán \\ Pontificia Universidad Javeriana, Colombia \\ Andrea Dionelly Murillo Casas \\ Pontificia Universidad Javeriana, Colombia \\ Leiddy Jazmín Muñoz PeÑa \\ Pontificia Universidad Javeriana, Colombia
}

a Autora de correspondencia. Correo electrónico: abertolotto@javeriana.edu.co

Cómo citar: Berttoloto AM, Vargas Vaca YA, Guzmán PC, Murillo Casas AD, Muñoz Peña LJ. Factores asociados a hipoglucemia neonatal transitoria en recién nacidos sanos, en el Hospital Universitario San Ignacio, estudio de casos y controles. Univ Med. 2017;58(3):1-5. doi: https://doi.org/10.11144/Javer iana.umed58-2.fahn

\section{RESUMEN}

Objetivo: Determinar factores de riesgo asociados a hipoglucemia neonatal transitoria en recién nacidos sanos. Materiales y métodos: Estudio de casos y controles anidados en una cohorte retrospectiva. Análisis de regresión logística. Resultados: Se evaluaron las variables de interés reportadas en la literatura en 40 casos y 40 controles. Se encontró como factor protector aislado para hipoglucemia el contacto piel a piel, que fue menor en los recién nacidos por cesárea; sin embargo, en el modelo de regresión logística, solamente el requerir fórmula láctea, por ineficaz lactancia materna, mostró ser un factor de riesgo para esta enfermedad $(\mathrm{OR}=9)$. Conclusión: La ineficiente lactancia materna exclusiva es el mayor factor de riesgo para hipoglucemia neonatal transitoria en recién nacidos a término sanos, lo que ocasiona un aumento en el uso de fórmula láctea.

Palabras clave

hipoglucemia; recién nacidos; factores de riesgo.

\section{ABSTRACT}

Objective: To determinate risk factors associated to neonatal hypoglycemia in healthy newborns. Materials and methods: Casecontrol study nested in a retrospective cohort. Logistic regression analysis. Results: 40 cases and 40 controls were evaluated. The main risk factors according to the literature were reported. Skin to skin care was found as an isolated protective factor to develop neonatal hypoglycemia, although, the regression logistic analysis showed, that only the need to enhance feeding with formula was a risk factor to develop neonatal hypoglycemia 
$(\mathrm{OR}=9)$. Conclusion: Inadequate exclusive breastfeeding remains the major risk factor for transient neonatal hypoglycaemia in healthy term newborn, leading to increased use of formula.

Keywords

hypoglycemia; newborn; risk factors.

\section{Introducción}

Aunque existe controversia acerca de la definición de hipoglucemia neonatal, así como de la concentración de glucosa que determina el diagnóstico, actualmente se ha aceptado que las cantidades de glucosa central menores a $47 \mathrm{mg} /$ $\mathrm{dl}(<2,6 \mathrm{mmol} / \mathrm{l})$ deben ser usados para definir la hipoglucemia incluso si el recién nacido se encuentra asintomático [1].

La hipoglucemia neonatal transitoria, constituye una de las principales causas de morbilidad al nacimiento, y su frecuencia de aparición cambia dependiendo de los factores de riesgo prenatales y del recién nacido [1].

Una gran variedad de funciones celulares se altera en condiciones de deficiencia energética, como el mantenimiento de los gradientes iónicos, la liberación/recaptación de neurotransmisores, la regulación de la concentración intracelular de calcio y la función mitocondrial. Hay evidencia que señala la participación del glutamato como excitotoxina en la muerte neuronal hipoglucémica [2].

La deprivación de glucosa puede generar en el cerebro alteraciones progresivas de la función neurológica, como encefalopatía, convulsiones, discapacidad cognitiva y muerte [2]. Como consecuencia de un episodio hipoglucémico, puede ocurrir daño neuronal, debido a que el cerebro es muy dependiente del aporte sanguíneo de glucosa, y esta es su fuente de energía principal [2,3].

Las manifestaciones clínicas de la hipoglucemia son variadas: puede aparecer irritabilidad, llanto anormal, letargia, estupor, convulsiones, coma, hipoactividad, hiporreactividad, hipotonía, disminución de la succión, temblores, vómito, diaforesis, respiración irregular, polipnea, apnea o cianosis y muerte; sin embargo, en muchos casos pueden ser asintomáticas [1,3].

En niños con factores de riesgo la hipoglucemia neonatal es frecuente con incidencias descritas entre el $14,7 \%$ y el $832 \%$ [4]. Los neonatos con factores de riesgo conocido para hipoglucemia caen dentro de dos categorías:

1. Exceso de utilización de glucosa, lo cual incluye estados hiperinsulinémicos, infecciones, etc. [5].

2. Inadecuada producción o liberación del sustrato, que incluye reservas disminuidas [5].

Esta distinción es importante, ya que actualmente se recomienda monitorización de glucosa de rutina mediante glucometría únicamente a los neonatos con factores de riesgo [5]. Por otro lado, esta entidad también puede aparecer en recién nacidos sanos, sin factores de riesgo conocidos, con una frecuencia mucho menor (2\% al $5 \%)$ [6].

El recién nacido sano está definido como el neonato a término, con problemas menores de transición, que no incluye grupos poblacionales de pacientes prematuros, con factores de riesgo o con complicaciones durante su transición inmediata y mediata temprana a la vida extrauterina $[4,7]$.

Respecto a los recién nacidos sanos, sin factores de riesgo, se han descrito factores asociados a complicaciones posnatales, por ejemplo, la vía del parto por cesárea, que en Colombia es un procedimiento frecuente; la edad gestacional entre 37 y 39 semanas; edad materna, y paridad $[8,9,10,11]$.

En la actualidad, el pilar fundamental de alimentación recomendado en los recién nacidos sanos es la lactancia materna exclusiva, por lo que, como política del hospital, no se administra leche industrializada, excepto cuando hay un inadecuado aporte referido por la madre y evidenciado por el personal de salud.

Por lo anterior, es un reto para el personal de salud determinar cuáles son los factores asociados a la aparición de hipoglucemia neonatal transitoria en recién nacidos sanos, 
para implementar acciones que permitan un diagnóstico y tratamiento oportuno.

\section{Materiales y métodos}

Se realizó un estudio de casos y controles retrospectivo anidados en una cohorte en el Hospital Universitario San Ignacio de la ciudad de Bogotá, Colombia, entre diciembre del 2013 y diciembre del 2016.

Se definió como caso un recién nacido vivo, a término (entre 37 y 41 semanas), con adecuado peso para la edad gestacional, evaluado por pediatra o neonatólogo por el método de Ballard, sin factores de riesgo para hipoglucemia neonatal y con diagnóstico de hipoglucemia neonatal (glucemia central menor o igual de $47 \mathrm{mg} / \mathrm{dl}$ ), y como control, un recién nacido vivo, a término (entre 37 y 41 semanas), con adecuado peso para la edad gestacional, evaluado por pediatra o neonatólogo por el método de Ballard, sin factor de riesgo para hipoglucemia neonatal y sin diagnóstico de hipoglucemia neonatal nacido el mismo día del caso.

Con base en la revisión de la literatura, las variables evaluadas fueron: edad materna (en años cumplidos en el momento del parto), número de hijos vivos en el momento del parto, vía del parto, número de controles prenatales realizados, peso al nacer, edad gestacional (medida por el método de Ballard), contacto piel a piel, tipo de seguridad social de la madre (régimen especial, contributivo o subsidiado) y requerimiento de alimentación con leche industrializada durante la estancia en alojamiento conjunto.

El tamaño de muestra se calculó usando el programa Epidata 4.1, a partir de un OR estimado de 1,5. Con relación un caso, un control (1:1), con proporción de casos expuestos del $30 \%$, para un IC del $95 \%$ y un poder del $80 \%$.

Posteriormente, se realizó el análisis estadístico con el programa Stata13.1 y se caracterizaron las variables de interés. La regresión logística múltiple analizó la asociación de cada factor en el evento, buscando el mejor modelo.

\section{Resultados}

Se evaluaron 40 casos y 40 controles, con características generales similares en ambos grupos. Se encontró que el $45 \%$ de los casos y el $60 \%$ de los controles eran recién nacidos de sexo femenino. La media de edad materna en el momento del parto en los casos fue de 29 años y en el grupo control fue de 26,9 años.

El promedio de peso al nacer en los dos grupos no tuvo diferencias estadísticamente significativas $(p=0,06)$. Se encontró un peso promedio de $2947 \mathrm{~g}$ en los casos, y de $3086 \mathrm{~g}$ en los controles.

El $52 \%$ de los casos fueron el primer hijo, y el $47,5 \%$ de los controles tenía esta característica. No hubo diferencias significativas en la paridad entre los casos y los controles, ( $p=0,64)$.

Todos los pacientes con hipoglucemia neonatal transitoria fueron producto de embarazos con dos o más controles prenatales; mientras que encontramos que en dos controles no hubo ningún tipo de control prenatal. Esta diferencia, sin embargo, no fue estadísticamente significativa $(\mathrm{p}=0,1)$. El promedio de edad gestacional en ambos grupos fue de 38 semanas. La mayoría de los pacientes estudiados pertenece al régimen de seguridad social contributivo, $(90$ $\%$ de los casos y $92,5 \%$ de los controles) (tabla $1)$. 
Tabla 1.Características generales de la población estudiada

\begin{tabular}{|c|c|c|c|}
\hline Variables & $\begin{array}{l}\text { Casos (hipoglucemia) } \\
\qquad \mathrm{n}=\mathbf{4 0}\end{array}$ & $\begin{array}{l}\text { Controles (sanos) } \\
\qquad \begin{array}{l}n=40\end{array}\end{array}$ & p \\
\hline $\begin{array}{l}\text { Edad materna (media en años } \\
\text { cumplidos) }\end{array}$ & 29,3 & 26,9 & 0,130 \\
\hline Peso al nacer (en gramos) & 2947 & 3086 & 0,060 \\
\hline Paridad materna & Piel a piel & & 0,650 \\
\hline Ningún hijo previo & 21 & 19 & \\
\hline Multípara & 19 & 21 & \\
\hline \multicolumn{3}{|c|}{ Via del parto } & 0,640 \\
\hline Vaginal & 24 & 26 & \\
\hline Cesárea & 16 & 14 & \\
\hline \multicolumn{3}{|c|}{ Controles prenatales (CPN) } & 0,100 \\
\hline $1 \mathrm{CPN}$ & 0 & 2 & \\
\hline $2 \mathrm{a} 4 \mathrm{CPN}$ & 11 & 16 & \\
\hline 5 o más CPN & 29 & 22 & \\
\hline $\begin{array}{l}\text { Edad Gestacional (media en } \\
\text { semanas) }\end{array}$ & 38,2 & 38,9 & \\
\hline \multicolumn{4}{|c|}{ Edad gestacional } \\
\hline 37 semanas & 9 & 1 & \\
\hline 38 semanas & 15 & 12 & \\
\hline 39 semanas & 12 & 17 & \\
\hline 40 semanas & 4 & 10 & \\
\hline \multicolumn{3}{|c|}{ Contacto piel a piel } & 0,019 \\
\hline No & 19 & 9 & \\
\hline Sí & 21 & 31 & \\
\hline
\end{tabular}

Se evaluó si había asociación entre hipoglucemia y las variables categóricas analizadas mediante la prueba de chi cuadrado y se encontró que solamente la lactancia materna exclusiva y el contacto piel a piel presentaba diferencias estadísticamente significativas con ( $\mathrm{p}$ $=0,002$ y 0,019 , respectivamente).

El $95 \%$ de los controles y solo el 67,5\% de los casos recibieron lactancia materna exclusiva y el $77 \%$ de los controles y solo el $52 \%$ de los casos tuvieron contacto piel a piel.

Se verificó mediante la prueba de Spearman que no había colinealidad entre las variables analizadas, excepto pacientes que nacieron por cesárea, quienes tuvieron menor contacto piel a piel, con una correlación de 0,7.

Llamó la atención que en los datos analizados la correlación entre peso al nacer y edad gestacional fue solo de 0,44 .

Las variables vía del parto y contacto piel a piel no generaron confusión en el modelo. Una regresión logística incorporó al modelo las variables que mostraban asociación y encontró que el contacto piel a piel es menor en pacientes producto de cesárea y de forma aislada es un factor protector para hipoglucemia neonatal transitoria $(\mathrm{OR}=0,32[0,12-0,83] ; \mathrm{p}=0,01)$. A pesar de lo anterior, al correr el modelo de regresión logística incorporando las variables que aportan al desenlace, el uso de fórmula láctea por ineficiente lactancia materna exclusiva sigue siendo el mayor factor de riesgo para hipoglucemia neonatal transitoria $(\mathrm{OR}=9 ; \mathrm{p}=$ 0,0016).

\section{Discusión}

Aunque el mecanismo de regulación de la glucosa en los recién nacidos ha sido objeto de profundos estudios, hacer un diagnóstico oportuno y un tratamiento adecuado en los recién nacidos sanos con hipoglucemia neonatal transitoria es un reto para el personal de salud, considerando que esta entidad, que en ocasiones es asintomática, puede generar daños irreversibles en el cerebro $[1,2,3,12]$.

Se conoce cuáles son los factores de riesgo tanto maternos como del recién nacido para este tipo de trastornos metabólicos, y hay lineamientos claros para el abordaje diagnóstico y terapéutico en este grupo de pacientes; sin embargo, esto no sucede en el grupo de neonatos considerados sanos $[4,5]$.

El hospital en el cual se realizó el estudio está certificado dentro del grupo de Instituciones Amigas de la Mujer y de la Infancia [13], por lo que se ofrece al recién nacido sano lactancia materna exclusiva, y solo en caso de que la madre dude y el personal de salud verifique que la lactancia no es suficiente, se administra fórmula láctea.

Aunque en la literatura se mencionan varios factores asociados a la aparición de hipoglucemia neonatal transitoria en los recién nacidos sanos, en el presente estudio solo se documentó que los niños producto de cesárea tenían menor contacto piel a piel con la madre en el posparto inmediato, por lo que es importante aclarar que aunque dicho contacto es, de forma aislada, un factor protector para hipoglucemia neonatal transitoria, esto no modificó el impacto del uso de fórmula láctea $[8,9,10,11]$. 
De acuerdo con los resultados del presente estudio, en nuestro medio la sospecha clínica de fallo en la lactancia materna exclusiva en el recién nacido sano y, por ende, el uso de fórmula láctea es el único factor de riesgo que impacta en el diagnóstico de hipoglucemia neonatal transitoria. $(\mathrm{OR}=9 ; \mathrm{p}=0,0016)$.

\section{Conclusiones}

La mayoría de los recién nacidos sanos que reciben lactancia materna exclusiva van a tener una evolución adecuada. Sin embargo, si la madre duda y el personal de salud considera que es preciso reforzar con fórmula láctea, se debe considerar que estos neonatos tienen nueve veces más riesgo de presentar hipoglucemia neonatal transitoria. La ineficiente lactancia materna exclusiva es el mayor factor de riesgo para hipoglucemia neonatal transitoria en recién nacidos a término sanos, lo que ocasiona un aumento en el uso de fórmula láctea.

\section{Referencias}

1. Adamkin DH. Neonatal hypoglycemia. Curr Opin Pediatr. 2016;28:150-5.

2. Blanco L, Massieu-Trigo L. Mecanismos de muerte neuronal asociados a la hipoglucemia. Arch Neurocien (Mex). 2005;10(2):83-91.

3. Courtney B, Grayson S, Mark P. Management strategies for neonatal hypoglycemia. J Pediatr Pharmacol Ther. 2013;18:3.

4. Harris DI, Weston PJ. Incidence of neonatal hypoglycemia in babies identified as at risk. J Pediatrics. 2012;161:5.

5. Wight N, Marinelli KA, The Academy of Breastfeeding Medicine. ABM Clinical Protocol \#1: Guidelines for blood glucose monitoring and treatment of hypoglycemia in term and late-preterm neonates. Breastfeed Med. 2014;9(4).

6. Hawdon JM. Neonatal hypoglycemia: are evidence-based clinical guidelines achievable? Neoreviews. 2014;15:e91.
7. Ministerio de Salud y Protección Social de Colombia. Guía de práctica clínica del recién nacido sano. Bogotá: Colciencias; 2013.

8. Armadans M, Ossorio MF, Pedicone C, Durán P, Ferrero F. Morbilidad en recién nacidos a término en relación a su edad gestacional. Rev Chil Pediatr. 2010;81(5):402-8.

9. Brown HK, Nixon K. Neonatal morbidity associated with late preterm and early term birth: the roles of gestational age and biological determinants of preterm birth. Int J Epidemiol. 2014;43:802-14.

10. Zhang X, Kramer MS. Variations in mortality and morbidity by gestational age among infants born at term. J. Pediatr. 2009 Mar;154(3):358-62.

11. Integrantes del Consenso de la Federación Colombiana de Obstetricia y Ginecología (Fecolsog) y la Federación Colombiana de Perinatología (Fecopen). Racionalización del uso de la cesárea en Colombia. Consenso de la Federación Colombiana de Obstetricia y Ginecología (FECOLSOG) y la Federación Colombiana de Perinatología (FECOPEN). Bogotá, 2014. Rev Colomb Obstetr Ginecol. 2014;65(2):139-151.

12. Pertierra A, Iglesias I. Hipoglucemia neonatal. An Pediatr Contin. 2013;11(3):142-51.

13. Unicef. Iniciativa Instituciones Amigas de la Mujer y la Infancia en el marco de los derechos: manual para su aplicación [internet]. Bogotá; 2005. Disponible en: https://www.unicef.org/colo mbia/pdf/IAMI-1.pdf 